Article

\title{
Fast $\gamma$-ray Variability: A Common Feature and Powerful Probe for Jetted AGNs
}

\author{
Neng-hui Liao \\ Key Laboratory of Dark Matter and Space Astronomy, Purple Mountain Observatory, \\ Chinese Academy of Sciences, Nanjing 210008, China; liaonh@pmo.ac.cn
}

Received: 17 May 2018; Accepted: 28 June 2018; Published: 3 July 2018

\begin{abstract}
Among the highly dynamical non-thermal universe, flashes of $\gamma$-ray photons from jets in active galactic nuclei (AGNs) are always attractive. Not only are they extraordinary observational phenomena, but they also become powerful probes of the jets. Benefiting from the current advanced $\gamma$-ray observational facilities, especially the Large Area Telescope aboard the Fermi space $\gamma$-ray observatory and Imaging Atmospheric Cherenkov Telescope arrays, fast (intraday) $\gamma$-ray variability has become a common feature detected in various subtypes of jetted AGNs. Moreover, extreme events with variability timescales down to a few minutes have been occasionally detected, which put a severe constraint on the classical jet model. Herein, recent studies on the detection of fast $\gamma$-ray variability in jetted AGNs are summarized, and corresponding implications are discussed. Scenarios proposed to explain the minute-scale $\gamma$-ray variability as well as future observational opportunities are also briefly summarized.
\end{abstract}

Keywords: galaxies: active; galaxies: jets; gamma rays: general; radiation mechanisms: non-thermal

\section{Introduction}

It is widely accepted that relativistic jets in active galactic nuclei (AGNs) play a crucial role in the nonthermal universe. Except for a few nearby star-forming galaxies, jetted AGNs are the dominant population of the extragalactic $\gamma$-ray sky (e.g., [1]). The majority of these $\gamma$-ray sources are blazars [2], including flat spectrum radio quasars (FSRQs) and BL Lacertae objects (BL Lacs), whose jets are closely aligned to our line of sight [3]. Due to significant relativistic beaming effects, they are characterized by their luminous and highly variable non-thermal continuum emissions from radio-to- $\gamma$-ray ( $\mathrm{GeV}$ and $\mathrm{TeV}$ ) energies, along with polarized radio and optical emissions [4,5]. On the other hand, jetted AGNs with relatively large jet inclination angles—-the so-called misaligned AGNs (i.e., radio galaxies and steep spectrum radio quasars, or SSRQs) —are also visible in the $\gamma$-ray sky [6,7]. In addition, the Large Area Telescope (LAT, [8]) aboard the Fermi space $\gamma$-ray observatory reveals radio-loud Narrow line Seyfert 1 galaxies (NLS1s) as a new subclass of $\gamma$-ray AGNs [9,10]. The spectral energy distribution of these jetted AGNs generally exhibits a two-bump structure in $\log v \mathrm{~F} v-\log v$ representation, where one is believed to be synchrotron emission of relativistic electrons based on the polarized radio and optical emissions, while the other peaking in the $\gamma$-ray domain is usually explained as inverse Compton scattering of soft photons by the same population of electrons [11]. The soft photons could be from the jet itself (i.e., the synchrotron emission), the synchrotron self-Compton (SSC) model [12]. The soft photons could also be from photons outside the jet, the external Compton (EC) models: including the UV emission from the accretion disk radiation [13], photons of emission lines from the broad-line region (BLR [14]), and the infrared emission from the dust torus [15]. Alternatively, the $\gamma$ rays could also be explained by hadronic scenarios [16]. According to their SED properties (i.e., the peak frequencies of the synchrotron bump), these sources are traditionally divided into 
low-synchrotron-peaked sources $\left(v_{\text {peak }}^{S}<10^{14} \mathrm{~Hz}\right)$, intermediate-synchrotron-peaked sources $\left(10^{14} \mathrm{~Hz}\right.$ $\left.<v_{\text {peak }}^{S}<10^{15} \mathrm{~Hz}\right)$, and high-synchrotron-peaked sources $\left(v_{\text {peak }}^{S}>10^{15} \mathrm{~Hz}\right)[2]$.

\section{Fast $\gamma$-ray Variability as a Common Feature Observed from Jetted AGNs}

One of the most extraordinary phenomena in jetted AGNs is their fast $\gamma$-ray variability (i.e., intraday variability), which provides a strong probe into the physical processes of the innermost regions of AGN jets. Benefiting from powerful observational facilities in the $\gamma$-ray domain, this phenomenon has been detected in different subtypes of jetted AGNs. Current understandings are provided below.

\subsection{Blazars}

Mrk 421, a high-frequency peaked BL Lac (HBL, [17]), is the first one with sub-hour very high energy (VHE) $\gamma$-ray variability detected by Whipple Observatory [18]. A more extreme phenomenon where the VHE $\gamma$-ray flux doubling time was as short as a few minutes has been observed from two other bright HBLs, PKS 2155-304 (2 min, [19]) and Mrk 501 (3 min, [20]), by H.E.S.S. and MAGIC, respectively. Since strong flares where these photons from "a hot hell" [21] are detected are highly unpredictable, together with the limited field of view of the current generation of Imaging Atmospheric Cherenkov Telescope arrays, such a detection requires not only the right telescope, but also the luck of pointing it in the right direction at the right time. So far, there has only been one other known detection of minute-timescale $\gamma$-ray variability from BL Lacs: BL Lacertae (10 $\mathrm{min}$, [22]) which is the prototype of its kind and a low-frequency-peaked BL Lac. Nevertheless, intraday VHE $\gamma$-ray variability has also been detected for the BL Lacs by ground $\gamma$-ray observational facilities [23].

It was a surprise when an ultrafast VHE $\gamma$-ray variability event was detected from a FSRQ: PKS $1222+21$ (10 min, [24]). Since the $\gamma$-ray spectra of FSRQs are generally softer than those of BL Lacs and due to the possibly severe absorption of the VHE $\gamma$-ray photons for interaction with soft photons (i.e., the extragalactic background light (EBL) and photons from the accretion system), there are only a few known VHE FSRQs so far. Searching for fast $\gamma$-ray variability of the former kind in the GeV domain to which the space-borne $\gamma$-ray observatories are sensitive is preferred. Actually, sub-day GeV $\gamma$-ray flux variations have been found in a few sources since the Compton Gamma Ray Observatory era [25]. Thanks to the Fermi-LAT, the number of such detections has significantly increased-see [26-29]; also see similar detections in the VHE regime [30]. However, due to its routine survey observation mode, the minimum variability timescale detected by Fermi-LAT is usually limited to a few hours. Interestingly, benefiting from a target of opportunity (ToO) repointing of Fermi-LAT, extremely fast $\mathrm{GeV} \gamma$-ray flux variability with a doubling time of less than 5 minutes that is comparable with those VHE $\gamma$-ray detections has been detected in a giant outburst of 3C 279 [31]. It is the second detection of minute-timescale $\gamma$-ray variability for FSRQs, and the first one in the $\mathrm{GeV}$ regime. Meanwhile, a third case has been detected by Fermi-LAT: a variability timescale down to $\sim 5$ min for CTA 102 in April 2017 [32]. All of these observational results strongly suggest that this extreme phenomenon is not rare among FSRQs.

Blazars could be bright beacons at early cosmic time, harboring super-massive black holes (SMBHs) heavier than one billion solar masses [33]. Studies of high-redshift blazars not only shed light on the formation and growth of the first generation of SMBHs and the role that relativistic jets play at that time, but also provide information on whether and how the jet properties change with cosmic time [34], along with their potential impacts on the evolution of AGNs and their host galaxies [35,36]. The farthest known $\gamma$-ray blazar detected by Fermi-LAT to date is NVSS J151002+570243 $(z=4.3$, [37]), along with six other sources with redshifts over 3 [38]. Studies of blazars with redshifts between 2 and 3 reveal their fast $\gamma$-ray variability $(\sim 2-3 \mathrm{~h}$ in the source frame, [39-41]). Moreover, a systematical temporal analysis of Fermi-LAT data of the seven sources beyond redshift 3 has been performed [42]. Significant long-term $\gamma$-ray variability has been found 
from five sources in monthly $\gamma$-ray light curves, in which three of them were reported for the first time. Furthermore, intraday $\gamma$-ray variations were detected from NVSS J053954-283956 $(z=3.1)$ and NVSS J080518+614423 $(z=3.0)$. The doubling variability timescale of the former source is limited to as short as $\lesssim 1 \mathrm{~h}$ (in the source frame). Together with variability amplitude over one order of magnitude (also see [43]), NVSS J053954-283956 is the most distant $\gamma$-ray flaring blazar so far (see Figure 1). Interestingly, the intraday optical variability of NVSS J163547+362930 $(z=3.6)$ was found based on archival Palomar Transient Factory (PTF)/intermediate PTF (iPTF) light curve. Since the variation of the optical emission of FSRQs has generally been observed simultaneously with the variation of their $\gamma$-ray emissions, fast $\gamma$-ray variability of NVSS J163547+362930 could soon be detected by Fermi-LAT.
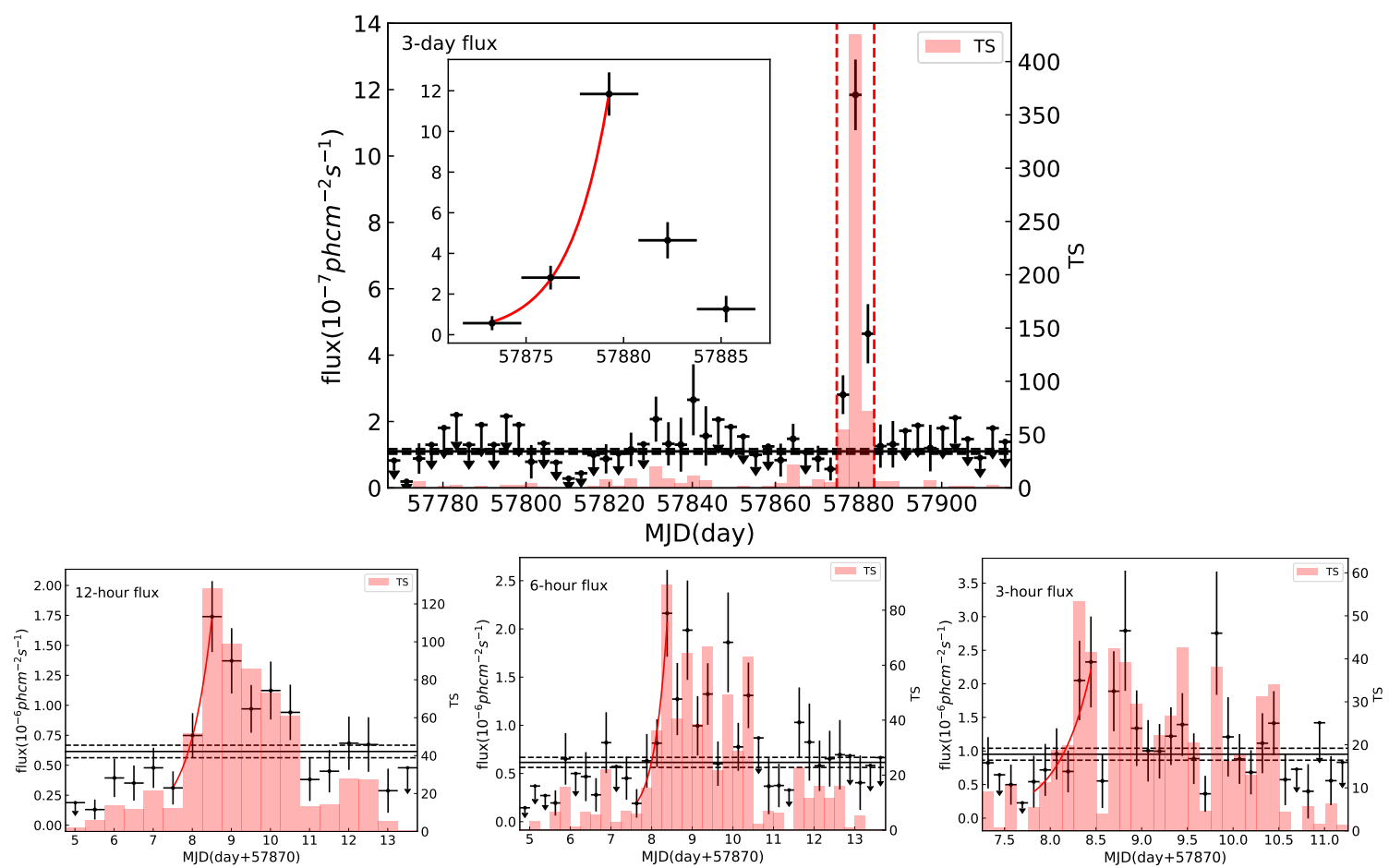

Figure 1. Fermi-Large Area Telescope (LAT) $\gamma$-ray light curves of NVSS J053954 - 283956 corresponding to the flare from which the fast variability has been detected. The horizontal solid line along with two dashed lines in each panel represent the average flux and its $1 \sigma$ error range. The red histogram represents the TS value in each time bin. Red solid lines are exponential fits of the ascent phase of this flare. (Top) The 3-day time bin light curve, in which the flare is marked by two vertical red lines; (Bottom) Zoomed-in intraday $\gamma$-ray light curves from which the doubling timescale is constrained as short as one hour (in the source frame). This figure can be also seen in Li et al. (2018) [42].

\subsection{Radio-Loud Narrow Line Seyfert I Galaxies}

NLS1s are a peculiar subclass of AGNs. They are believed to be in an early stage of AGN activity because of their small black hole (BH) masses ${ }^{1}$ ( between $10^{5}$ and $10^{8} \mathbf{M}_{\odot}$ ) and accretion systems with high Eddington ratios ( $\gtrsim 0.1$ ) which are different from normal jetted AGNs (e.g., blazars) [45]. Therefore, they serve as an intriguing population of sources, shedding important new insights into the formation of AGN jets under extreme conditions. Among a handful of $\gamma$-ray radio-loud NLS1s, fast $\gamma$-ray variability on a timescale of $\sim 3 \mathrm{~h}$ has been detected from $1 \mathrm{H} 0323+342$ [46] ( also see a hint of a similar behavior in PKS 1502+036 [47]).

1 The "intrinsic" BH mass of the NLSy1 is still under debate [44]. 


\subsection{Radio Intermediate Quasar: III Zw 2}

In early time, a bimodal distribution of radio properties from optical-selected quasars has been found [48], and radio-loudness (i.e., ratio of the radio flux at $5 \mathrm{GHz}$ to the optical flux in the $\boldsymbol{B}$-band) is usually adopted as a convenient tool for classification. However, based on recent deep and massive radio and optical surveys, such a bimodal distribution is not preferred [49]. Beyond the classical radio-loud and radio-quiet quasars, there is another population, the so-called "radio-intermediate quasar" (RIQ, e.g., [50]). III Zw 2, the prototype of this population, on one hand shows typical blazar behaviors including a detection of superluminal jet motion as well as violent radio variability. On the other hand, unlike blazars, its radio-loudness is moderate and its extended radio emission is very weak compared with its core emission [51].

In order to probe the connection between III Zw 2 and blazars, we have carried out a thorough investigation of its multiwavelength data from radio to $\gamma$-ray regimes [29]. Particularly, two distinct violent $\gamma$-ray flares have been detected by Fermi-LAT, which suggests III Zw 2 is a strong $\gamma$-ray emitter, though no significant signal has been found in the time-averaged 7-year data. Simultaneous optical and $\gamma$-ray variations have been observed in one flare, which is helpful to identify the association. Moreover, fast $\gamma$-ray variability with a doubling timescale of $2.5 \mathrm{~h}$ has been detected in another $\gamma$-ray flare, when a newly ejected jet knot was detected by the MOJAVE program 50 days later (see Figure 2) [52]. Note that III Zw 2 is the first known $\gamma$-ray RIQ so far, and even for FSRQs, a minimum $\gamma$-ray variability timescale of $\sim 1-2 \mathrm{~h}$ is extreme.
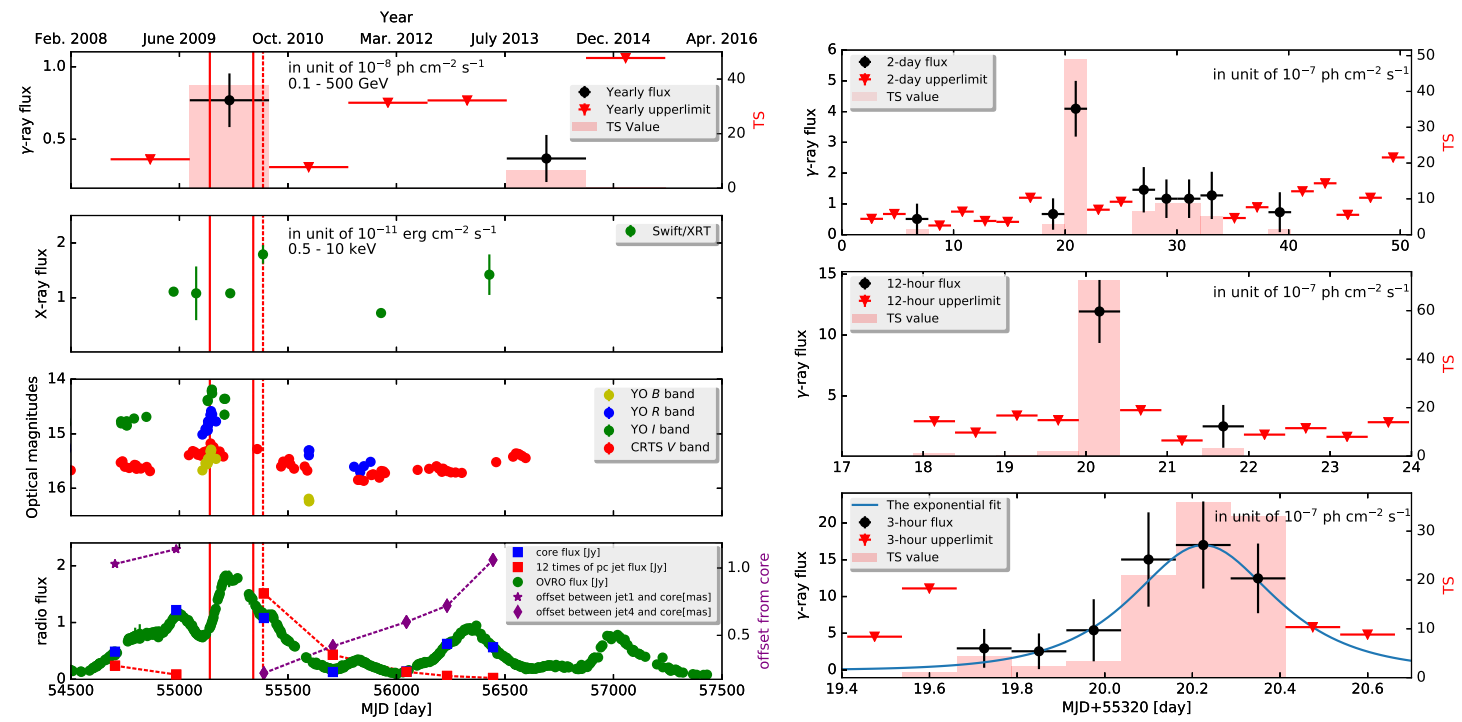

Figure 2. (Left) The multiwavelength light curves of III Zw 2. In the $\gamma$-ray (Fermi-LAT) panel, the black circles correspond to the fluxes and the red triangles are the $2 \sigma$ upper limits. The red bars are the TS values in each data bin. In the optical panel, green, blue, and yellow circles are the magnitudes of $I$, $\boldsymbol{R}$, and $\boldsymbol{B}$ bands observed in Yunnan Observatories, respectively. The red circles are CRTS $\boldsymbol{V}$ band magnitudes minus $0.8 \mathrm{mag}$ (for more details see Section 2.3 in Liao et al. (2016) [29]). In the radio panel, the green circles are OVRO single-dish flux densities, and the blue and purple circles are the MOJAVE core and parsec jet VLBA flux densities. The two solid red vertical lines mark the peaking times of the two $\gamma$-ray flares and the dashed red vertical line corresponds to the time of the simultaneous Planck, Swift, and Fermi campaign. (Right) Zoomed-in Fermi-LAT $\gamma$-ray light curves for the $\gamma$-ray flare on May 2010, including the 2-day bin (upper panel), 12-h bin (middle panel), and 3-h (bottom panel) light curves. A exponential fit of this intense flare is also shown along with the 3 -h light curve. This figure can be also seen in Liao et al. (2016) [29]. 


\subsection{Misaligned AGNs}

Due to their relatively large jet inclination angles (i.e., $\gtrsim \mathbf{1 0}^{\circ}$ ), the Doppler beaming effect should not be significant, and misaligned AGNs tend to have milder $\gamma$-ray variability (usually on timescales of months) and slower apparent knot speeds than blazars. Unexpectedly, fast VHE $\gamma$-ray variability on timescales of $\sim 1$ day was detected from the nearby radio galaxy M87 by H.E.S.S. [53]. Another case is IC 310, from which MAGIC observations reveal an ultrafast VHE $\gamma$-ray variability with doubling timescales shorter than 5 min [54]. However, IC 310 exhibits a transitional behavior between a radio galaxy and a BL Lac, which makes its classification still under debate. Recently, NGC 1275 has become the third case based on its hour-scale $\mathrm{GeV} \gamma$-ray variability detected by Fermi-LAT [55]. Meanwhile, night-to-night VHE $\gamma$-ray variability of NGC 1275 has also been seen by MAGIC [56].

\section{Implications and Prospects}

The observed minimum $\gamma$-ray variability timescale naturally reflects the radius of $\gamma$-ray radiation region,

$$
R_{\gamma}^{\prime}=c t_{v a r}^{\prime} \lesssim \delta c t_{v a r, o b s}(1+z)^{-1},
$$

where $\delta$ is the Doppler factor and $c$ is the speed of light. Therefore, fast $\gamma$-ray variability indicates a compact radiation region. However, when the radius of this compact region is compared with the gravitational radius of the central $\mathrm{SMBH}$, there are large uncertainties in the estimations of the Doppler factor as well as the BH mass. To avoid heavy absorption on $\gamma$ rays from soft photons via $\gamma \gamma$ process, the optical depth should not be high,

$$
\tau_{\gamma \gamma}\left(x^{\prime}\right)=\frac{\sigma_{\mathrm{T}}}{5} n^{\prime}\left(x_{\mathfrak{t}}^{\prime}\right) x_{\mathfrak{t}}^{\prime} R^{\prime} \lesssim 1,
$$

where $\sigma_{\mathrm{T}}$ is the scattering Thomson cross section, $n^{\prime}\left(x^{\prime}\right)$ is the differential comoving number density of the target photon per energy, $x_{\mathfrak{t}}^{\prime}$ is the energy of the target photon in dimensionless units, and $\boldsymbol{R}^{\prime}$ is the absorption length [57]. Either the jet radiation itself and/or external emissions (e.g., from accretion disk or broad emission lines) can serve as the absorbing soft photons. Alternatively, the absorption from the EBL could be important, especially for the high redshift sources [58]. Meanwhile, it is reasonable to assume that the minimum variability timescale is a good approximation of the radiative cooling timescale [59],

$$
t_{\text {cool }}^{\prime}\left(\gamma_{b r}\right) \simeq 3 m_{e} c /\left(4 \sigma_{T} \gamma_{b r} u_{c o o l}^{\prime}\right) \simeq t_{v a r}^{\prime}
$$

where $\gamma_{b r}$ corresponds to cooling break energy in the electron distribution. $\gamma_{b r}$ can be estimated as $\sqrt{\left(v_{S S C, b r} / v_{s y n, b r}\right)}$ in the synchrotron self-Compton (SSC) model for HBLs and $\sqrt{\left(v_{E C, b r} / v_{e x t, b r} / \delta^{2}\right)}$ in the external Compton (EC) model for FSRQs. $v_{s y n, b r}, v_{S S C, b r}, v_{E C, b r}$, and $v_{e x t, b r}$ correspond to the peaking frequencies of synchrotron, SSC, EC, and external soft photon emissions, respectively. The cooling photon density $\boldsymbol{u}_{\text {cool }}^{\prime}$ can also be obtained from the observed synchrotron luminosity or the external emissions (e.g., the accretion disk emission), respectively. Note that this formula can only applied within the Thomson regime (for the Klein-Nishina regime, see e.g., [60]). Based on these two approaches, one can provide lower limits of the Doppler factor, which is a crucial quantity of AGN jets. Such a constraint could be rather severe when applied to the detection of minute-scale $\gamma$-ray variability, $\delta \gtrsim \mathbf{5 0}$. It is significantly higher than the typical value given by the kinetic radio observations, $\lesssim \mathbf{1 0}$. In the classical one-zone jet model for a conical geometry, distance scale between the $\gamma$-ray radiation region and the central SMBHs, $r_{\gamma}$, is proportional to the size of the radiation region,

$$
r_{\gamma} \simeq R_{\gamma} / \theta \simeq R_{\gamma} \Gamma \simeq R_{\gamma} \delta,
$$

where $\boldsymbol{\theta}$ is the jet opening angle and $\boldsymbol{\Gamma}$ is the jet bulk Lorentz factor, and thus a compact radiation region should be deeply embedded in the radiation field of the broad line region. Note that radio 
observations suggest a causal jet $(\boldsymbol{\Gamma} \boldsymbol{\theta} \sim 0.2[61])$ rather than the assumption $\boldsymbol{\Gamma} \boldsymbol{\theta}=1$ adopted in the above formula. Detections of ultrafast $\gamma$-ray variability among FSRQs put a strong challenge to this standard picture, since in this case $\gamma$ rays would be heavily absorbed from the intense external photons. For high-redshift blazars, detections of their fast $\gamma$-ray variability should not be a surprise because highly beamed sources are more likely to be seen there due to the Malmquist bias. However, based on these detections, no significant difference of the Doppler factor between high-redshift blazars and nearby ones is found. Therefore, SDSS-FIRST detected jetted AGN with $z>3$, which is fairly less than the expectation according to the Swift-BAT-detected high-redshift blazars [35], is likely due to selection effects rather than a result of a decrease of the average bulk Lorentz factor at higher redshifts. For the radio-loud NLS1s and RIQ III Zw 2, the detected fast $\gamma$-ray variability provides firm evidence that their central engine resembles that of blazars. Considering that the radio core of III Zw 2 is similar to those of young radio sources, along with a faint relic of the distant radio lobe, one possibility is that the jet has recently been re-triggered. Since III Zw 2 is hosted by a merging galaxy and the galaxy merger phenomenon is believed to be a possible path for generating radio-loud AGNs, III Zw 2 could be an interesting target that provides crucial information of the early activity of AGN jets as well as their triggering mechanisms. Finally, detections of fast $\gamma$-ray variability from misaligned AGNs are quite appealing. Since the Doppler beaming effects are modest for misaligned AGNs and hence the scenarios which work for blazars should not be applied to misaligned AGNs, they offer a novel perspective to approach this intense high-energy phenomenon.

Several scenarios were proposed soon after the detections of minute-scale $\gamma$-ray variability (for a recent summary see, e.g., [62]). Firstly, flares could be initiated by relativistically moving sub-regions through the jet material, the so-called "jets in a jet" [63,64]. Interaction between the jet and external materials (i.e., a star or cloud [65]) may bring such mini-jets where particles are accelerated by the magnetic reconnection process [66]. Alternatively, by analogy with the magnetospheric model usually adopted for radio-loud pulsars, charged particles are accelerated by unscreened electric fields in a charge-starved vacuum gap of the SMBH magnetosphere [67]. The advantage of this magnetospheric scenario is that it could work beyond blazars (e.g., misaligned AGNs). Due to the lack of Doppler boosting, the $\gamma$-ray luminosity predicted in this scenario should be quite modest when compared to blazars. However, note that the peaking luminosity of the $\gamma$-ray flare of NGC 1275 from which the fast variability is detected reaches up to $\mathbf{1 0}^{\mathbf{4 4}} \mathrm{erg} \mathrm{s}^{-1}$ (in the proper frame, assuming $\delta=4$, [55]).

The physical processes behind the ultrafast $\gamma$-ray variability are still poorly known. What is the connection between this phenomenon and the fundamental processes of AGN jets? For example, it is suggested to be happening at a highly magnetized place that is likely close to the jet base. Therefore, is there a link between the ultrafast $\gamma$-ray variability and the jet launching process? Meanwhile, is the triggering mechanism different among diverse subtypes of jetted AGNs (e.g., between FSRQs and BL Lacs)? Or does the triggering mechanism correspond to some basic magnetohydrodynamic instabilities which might be irrelevant for the jets? Note that there have only been seven detections of minute-scale $\gamma$-ray variability for jetted AGNs so far, and the majority of these were detected in the $\mathrm{TeV}$ domain. The next generation of ground $\gamma$-ray facility, the Cherenkov Telescope Array [68], which has a lower energy threshold, wider field of view, and significantly enhanced detection sensitivity, accompanied with $\gamma$-ray sky alerts $[69,70]$, will be helpful in answering these questions.

Funding: Neng-hui Liao is supported by National Natural Science Foundation of China under grant 11703093.

Acknowledgments: Neng-hui Liao appreciates helpful suggestions from the anonymous referees.

Conflicts of Interest: The author declares no conflict of interest. 


\section{References}

1. Acero, F.; Ackermann, M.; Ajello, M.; Albert, A.; Atwood, W.B.; Axelsson, M.; Baldini, L.; Ballet, J.; Barbiellini, G.; Bastieri, D.; et al. Fermi large area telescope third source catalog. Astrophys. J. Suppl. 2015, 218, 280-286. [CrossRef]

2. Ackermann, M.; Ajello, M.; Atwood, W.B.; Baldini, L.; Ballet, J.; Barbiellini, G.; Bastieri, D.; Gonzalez, B.J.; Bellazzini, R. The Third Catalog of Active Galactic Nuclei Detected by the Fermi Large Area Telescope. Astrophys. J. 2015, 810, 14.

3. Blandford, R.D.; Rees, M.J. Some Comments on Radiation Mechanisms in Lacertids. In Proceedings of the Pittsburgh Conference on BL Lac Objects, Pittsburgh, PA, USA, 24-26 April 1978.

4. Ulrich, M.-H.; Maraschi, L.; Urry, C.M. Variability of Active Galactic Nuclei. Annu. Rev. Astron. Astrophys. 1997, 35, 445-502. [CrossRef]

5. Madejski, G.; Sikora, M. Gamma-Ray Observations of Active Galactic Nuclei. Annu. Rev. Astron. Astrophys. 2016, 54, 725-760. [CrossRef]

6. Abdo, A.A.; Ackermann, M.; Ajello, M.; Baldini, L.; Ballet, J.; Barbiellini, G.; Bastieri, D.; Bechtol, K.; Bellazzini, R.; Berenji, B.; et al. Fermi Large Area Telescope Observations of Misaligned Active Galactic Nuclei. Astrophys. J. 2010, 720, 912-922. [CrossRef]

7. Liao, N.-H.; Xin, Y.-L.; Li, S.; Jiang, W.; Liang, Y.-F.; Li, X.; Zhang, P.-F.; Chen, L.; Bai, J.-M.; Fan, Y.-Z. Discovery of $\gamma$-ray Emission from the Strongly Lobe-dominated Quasar 3C 275.1. Astrophys. J. 2015, 808, 74. [CrossRef]

8. Atwood, W.B.; Abdo, A.A.; Ackermann, M.; Althouse, W.; Anderson, B.; Axelsson, M.; Baldini, L.; Ballet, J.; Band, D.L.; Barbiellini, B.; et al. The Large Area Telescope on the Fermi Gamma-Ray Space Telescope Mission. Astrophys. J. 2009, 697, 1071-1102. [CrossRef]

9. Abdo, A.A.; Ackermann, M.; Ajello, M.; Axelsson, M.; Baldini, L.; Ballet, J.; Barbiellini, G.; Bastieri, D.; Battelino, M.; Baughman, B.; et al. Fermi/Large Area Telescope Discovery of Gamma-Ray Emission from a Relativistic Jet in the Narrow-Line Quasar PMN J0948+0022. Astrophys. J. 2009, 699, 976-984. [CrossRef]

10. Abdo, A.A.; Ackermann, M.; Ajello, M.; Baldini, L.; Ballet, J.; Barbiellini, G.; Bastieri, D.; Bechtol, K.; Bellazzini, B. Radio-Loud Narrow-Line Seyfert 1 as a New Class of Gamma-Ray Active Galactic Nuclei. Astrophys. J. Lett. 2009, 707, L142-L147. [CrossRef]

11. Sikora, M.; Stawarz, Ł.; Moderski, R.; Nalewajko, K.; Madejski, G.M. Constraining Emission Models of Luminous Blazar Sources. Astrophys. J. 2009, 704, 38-50. [CrossRef]

12. Maraschi, L.; Ghisellini, G.; Celotti, A. A jet model for the gamma-ray emitting blazar 3C 279. Astrophys. J. Lett. 1992, 397, L5-L9. [CrossRef]

13. Dermer, C.D.; Schlickeiser, R. Model for the High-Energy Emission from Blazars. Astrophys. J. 1993, 416, 458. [CrossRef]

14. Sikora, M.; Begelman, M.C.; Rees, M.J. Comptonization of diffuse ambient radiation by a relativistic jet: The source of gamma rays from blazars? Astrophys. J. 1994, 421, 153-162. [CrossRef]

15. Błażejowski, M.; Sikora, M.; Moderski, R.; Madejski, G.M. Comptonization of Infrared Radiation from Hot Dust by Relativistic Jets in Quasars. Astrophys. J. 2000, 545, 107-116. [CrossRef]

16. Böttcher, M.; Reimer, A.; Sweeney, K.; Prakash, A. Leptonic and hadronic modeling of Fermi-detected blazars. Astrophys. J. 2013, 768, 54. [CrossRef]

17. Padovani, P.; Giommi, P. The connection between x-ray- and radio-selected BL Lacertae objects. Astrophys. J. 1995, 444, 567-581. [CrossRef]

18. Gaidos, J.A.; Akerlof, C.W.; Biller, S.; Boyle, P.J.; Breslin, A.C.; Buckley, J.H.; Carter-Lewis, D.A.; Catanese, M. Extremely rapid bursts of $\mathrm{TeV}$ photons from the active galaxy Markarian 421. Nature 1996, 383, 319-320. [CrossRef]

19. Aharonian, F.; Akhperjanian, A.G.; Bazer-Bachi, A.R.; Behera, B.; Beilicke, M.; Benbow, W.; Berge, D.; Bernlöhr, K.; Boisson, C.; et al. An Exceptional Very High Energy Gamma-Ray Flare of PKS 2155-304. Astrophys. J. Lett. 2007, 664, L71-L74. [CrossRef]

20. Albert, J.; Aliu, E.; Anderhub, H.; Antoranz, P.; Armada, A.; Baixeras, C.; Barrio, J.A.; Bartko, H.; Bastieri, C. Variable Very High Energy $\gamma$-Ray Emission from Markarian 501. Astrophys. J. 2007, 669, 862-883. [CrossRef]

21. Weekes, T. Astrophysics: Photons from a hotter hell. Nature 2007, 448, 760-762. [CrossRef] [PubMed] 
22. Arlen, T.; Aune, T.; Beilicke, M.; Benbow, W.; Bouvier, A.; Buckley, J.H.; Bugaev, V.; Cesarini, A.; Ciupik, L.; Connolly, M.P.; et al. Rapid TeV Gamma-Ray Flaring of BL Lacertae. Astrophys. J. 2013, 762, 92. [CrossRef]

23. Santander, M. The exceptional TeV flaring activity of the blazar 1ES 1959+650 in 2015 and 2016 as observed with VERITAS. arXiv 2017, arXiv:1709.02365.

24. Aleksić, J.; Antonelli, L.A.; Antoranz, P.; Backes, M.; Barrio, J.A.; Bastieri, D.; Becerra González, J. MAGIC Discovery of Very High Energy Emission from the FSRQ PKS 1222+21. Astrophys. J. Lett. 2011, 730, L8. [CrossRef]

25. Mattox, J.R.; Wagner, S. J.; Malkan, M.; McGlynn, T.A.; Schachter, J.F.; Grove, J.E.; Johnson, W. N.; Kurfess, J.D. An Intense Gamma-Ray Flare of PKS 1622-297. Astrophys. J. 1997, 476, 692-697. [CrossRef]

26. Foschini, L.; Ghisellini, G.; Tavecchio, F.; Bonnoli, G.; Stamerra, A. Search for the shortest variability at gamma rays in flat-spectrum radio quasars. Astron. Astrophys. 2011, 530, A77. [CrossRef]

27. Saito, S.; Stawarz, Ł.; Tanaka, Y.T.; Takahashi, T.; Madejski, G.; D'Ammando, F. Very Rapid High-amplitude Gamma-Ray Variability in Luminous Blazar PKS 1510-089 Studied with Fermi-LAT. Astrophys. J. Lett. 2013, 766, L11. [CrossRef]

28. Liao, N.H.; Bai, J.M. Rapid high-amplitude $\gamma$-ray variability in blazar PKS 0507+17. New Astron. 2015, 34, 134-138. [CrossRef]

29. Liao, N.-H.; Xin, Y.-L.; Fan, X.-L.; Weng, S.-S.; Li, S.-K.; Chen, L.; Fan, Y.-Z. Discovery of $\gamma$-Ray Emission from the Radio-intermediate Quasar III Zw 2: Violent Jet Activity with Intraday $\gamma$-Ray Variability. Astrophys. J. Suppl. 2016, 226, 17. [CrossRef]

30. Zacharias, M.; Sitarek, J.; Dominis Prester, D. The exceptional VHE gamma-ray outburst of PKS 1510-089 in May 2016. arXiv 2017, arXiv:1708.00653.

31. Ackermann, M.; Anantua, R.; Asano, K.; Baldini, L.; Barbiellini, G.; Bastieri, D.; Becerra Gonzalez, J.; Bellazzini, R. Minute-timescale $>100 \mathrm{MeV} \gamma$-Ray Variability during the Giant Outburst of Quasar 3C 279 Observed by Fermi-LAT in 2015 June. Astrophys. J. Lett. 2016, 824, L20. [CrossRef]

32. Shukla, A.; Mannheim, K.; Patel, S.R.; Roy, J.; Chitnis, V.R.; Dorner, D.; Rao, A.R.; Anupama, G.C.; Wendel, C. Short-timescale $\gamma$-Ray Variability in CTA 102. Astrophys. J. Lett. 2018, 854, L26. [CrossRef]

33. Ghisellini, G.; Della Ceca, R.; Volonteri, M.; Ghirlanda, G.; Tavecchio, F.; Foschini, L.; Tagliaferri, G.; Haardt, F.; Pareschi, G.; Grindlay, J. Chasing the heaviest black holes of jetted active galactic nuclei. Mon. Not. R. Astron. Soc. 2010, 405, 387-400. [CrossRef]

34. Volonteri, M. Formation of supermassive black holes. Astron. Astrophys. Rev. 2010, 18, 279-315. [CrossRef]

35. Volonteri, M.; Haardt, F.; Ghisellini, G.; Della Ceca, R. Blazars in the early Universe. Mon. Not. R. Astron. Soc. 2011, 416, 216-224. [CrossRef]

36. Fabian, A.C. Observational Evidence of Active Galactic Nuclei Feedback. Annu. Rev. Astron. Astrophys. 2012, 50, 455-489. [CrossRef]

37. Flesch, E.W. The Half Million Quasars (HMQ) Catalogue. Publ. Astron. Soc. Aust. 2015, 32, e010. [CrossRef]

38. Ackermann, M.; Ajello, M.; Baldini, L.; Ballet, J.; Barbiellini, G.; Bastieri, D.; Gonzalez, B.J.; Bellazzini, R. Gamma-Ray Blazars within the First 2 Billion Years. Astrophys. J. Lett. 2017, 837, L5. [CrossRef]

39. Akyuz, A.; Thompson, D. J.; Donato, D.; Perkins, J. S.; Fuhrmann, L.; Angelakis, E.; Zensus, J.A.; Larsson, S.; Sokolovsky, K.; Kurtanidze, O. Long-term multiwavelength studies of high-redshift blazar $0836+710$. Astron. Astrophys. 2013, 556, A71. [CrossRef]

40. Abdo, A.A.; Ackermann, M.; Ajello, M.; Allafort, A.; Amin, M.A.; Baldini, L.; Barbiellini, G.; Bastieri, D. Gamma-Ray Flaring Activity from the Gravitationally Lensed Blazar PKS 1830-211 Observed by Fermi LAT. Astrophys. J. 2015, 799, 143. [CrossRef]

41. D'Ammando, F.; Orienti, M. High-energy properties of the high-redshift flat spectrum radio quasar PKS 2149-306. Mon. Not. R. Astron. Soc. 2016, 455, 1881-1891. [CrossRef]

42. Li, S.; Xia, Z.-Q.; Liang, Y.-F.; Liao, N.-H.; Fan, Y.-Z. Fast $\gamma$-Ray Variability in Blazars beyond Redshift 3. Astrophys. J. 2018, 853, 159. [CrossRef]

43. Cheung, C.C. Fermi LAT detection of a GeV flare from High-redshift Blazar PKS 0537-286. Astronomer's Telegram 2017, No. 10356.

44. Calderone, G.; Ghisellini, G.; Colpi, M.; Dotti, M. Black hole mass estimate for a sample of radio-loud narrow-line Seyfert 1 galaxies. Mon. Not. R. Astron. Soc. 2013, 431, 210-239. [CrossRef]

45. Komossa, S. Narrow-line Seyfert 1 Galaxies. Rev. Mex. Astron. Astrofisica Conf. Ser. 2008, 32, 86-92. 
46. Paliya, V.S.; Sahayanathan, S.; Parker, M.L.; Fabian, A.C.; Stalin, C.S.; Anjum, A.; Pandey, S.B. The Peculiar Radio-loud Narrow Line Seyfert 1 Galaxy 1H 0323+342. Astrophys. J. 2014, 789, 143. [CrossRef]

47. D'Ammando, F.; Orienti, M.; Finke, J.; Hovatta, T.; Giroletti, M.; Max-Moerbeck, W.; Pearson, T.J.; Readhead, A.C.S.; Reeves, R.A.; Richards, J.L. The awakening of the $\gamma$-ray narrow-line Seyfert 1 galaxy PKS 1502+036. Mon. Not. R. Astron. Soc. 2016, 463, 4469-4480. [CrossRef]

48. Kellermann, K.I.; Sramek, R.; Schmidt, M.; Shaffer, D.B.; Green, R. VLA observations of objects in the Palomar Bright Quasar Survey. Astron. J. 1989, 98, 1195-1207. [CrossRef]

49. Ivezić, Ž.; Menou, K.; Knapp, G.R.; Strauss, M.A.; Lupton, R.H.; Vanden Berk, D.E.; Richards, G.T.; Tremonti, C.; Weinstein, M.A.; Anderson, S.; et al. Optical and Radio Properties of Extragalactic Sources Observed by the FIRST Survey and the Sloan Digital Sky Survey. Astron. J. 2002, 124, 2364-2400.

50. Falcke, H.; Patnaik, A.R.; Sherwood, W. EVN + MERLIN Observations of Radio-intermediate Quasars: Evidence for Boosted Radio-weak Quasars. Astrophys. J. Lett. 1996, 473, L13. [CrossRef]

51. Brunthaler, A.; Falcke, H.; Bower, G.C.; Aller, M.F.; Aller, H.D.; Teräsranta, H. The extreme flare in III Zw 2: Evolution of a radio jet in a Seyfert galaxy. Astron. Astrophys. 2005, 435, 497-506. [CrossRef]

52. Lister, M.L.; Aller, M.F.; Aller, H.D.; Homan, D.C.; Kellermann, K.I.; Kovalev, Y.Y.; Pushkarev, A.B.; Richards, J.L.; Ros, E.; Savolainen, T. MOJAVE: XIII. Parsec-scale AGN Jet Kinematics Analysis Based on 19 years of VLBA Observations at $15 \mathrm{GHz}$. Astrophys. J. 2016, 152, 12. [CrossRef]

53. Aharonian, F.; Akhperjanian, A.G.; Bazer-Bachi, A.R.; Beilicke, M.; Benbow, W.; Berge, D.; Bernlöhr, K.; Boisson, C.; Bolz, O. Fast Variability of Tera-Electron Volt $\gamma$ Rays from the Radio Galaxy M87. Science 2006, 314, 1424-1427. [CrossRef] [PubMed]

54. Aleksić, J.; Ansoldi, S.; Antonelli, L.A.; Antoranz, P.; Babic, A.; Bangale, P.; Barrio, J.A.; González, J.B.; Bednarek, W.; Bernardini, E.; et al. Black hole lightning due to particle acceleration at subhorizon scales. Science 2014, 346, 1080-1084. [CrossRef] [PubMed]

55. Baghmanyan, V.; Gasparyan, S.; Sahakyan, N. Rapid Gamma-Ray Variability of NGC 1275. Astrophys. J. 2017, 848, 111. [CrossRef]

56. Mirzoyan, R. MAGIC detection of a giant flaring activity from NGC 1275 at very-high-energy gamma rays. Astronomer's Telegram, 2017, No. 9929.

57. Dondi, L.; Ghisellini, G. Gamma-ray-loud blazars and beaming. Mon. Not. R. Astron. Soc. 1995, 273, 583-595. [CrossRef]

58. Abdo, A.A. Fermi Large Area Telescope Constraints on the Gamma-ray Opacity of the Universe. Astrophys. J. 2010, 723, 1082-1096. [CrossRef]

59. Begelman, M.C.; Fabian, A.C.; Rees, M.J. Implications of very rapid TeV variability in blazars. Mon. Not. R. Astron. Soc. 2008, 384, L19-L23. [CrossRef]

60. Moderski, R.; Sikora, M.; Coppi, P.S.; Aharonian, F. Klein-Nishina effects in the spectra of non-thermal sources immersed in external radiation fields. Mon. Not. R. Astron. Soc. 2005, 363, 954-966. [CrossRef]

61. Clausen-Brown, E.; Savolainen, T.; Pushkarev, A.B.; Kovalev, Y.Y.; Zensus, J.A. Causal connection in parsec-scale relativistic jets: Results from the MOJAVE VLBI survey. Astron. Astrophys. 2013, 558, A144. [CrossRef]

62. Aharonian, F.A.; Barkov, M.V.; Khangulyan, D. Scenarios for Ultrafast Gamma-Ray Variability in AGN. Astrophys. J. 2017, 841, 61. [CrossRef]

63. Ghisellini, G.; Tavecchio, F.; Bodo, G.; Celotti, A. TeV variability in blazars: How fast can it be? Mon. Not. R. Astron. Soc. 2009, 393, L16-L20. [CrossRef]

64. Giannios, D.; Uzdensky, D.A.; Begelman, M.C. Fast TeV variability in blazars: Jets in a jet. Mon. Not. R. Astron. Soc. 2009, 395, L29-L33. [CrossRef]

65. Barkov, M.V.; Aharonian, F.A.; Bogovalov, S.V.; Kelner, S.R.; Khangulyan, D. Rapid TeV Variability in Blazars as a Result of Jet-Star Interaction. Astrophys. J. 2012, 749, 119. [CrossRef]

66. Blandford, R.; Yuan, Y.; Hoshino, M.; Sironi, L. Magnetoluminescence. Space Sci. Rev. 2017, 207, $291-317$. [CrossRef]

67. Levinson, A.; Rieger, F. Variable TeV Emission as a Manifestation of Jet Formation in M87? Astrophys. J. 2011, 730, 123. [CrossRef]

68. Actis, M.; Agnetta, G.; Aharonian, F.; Akhperjanian, A.; Aleksić, J.; Aliu, E.; Allan, D.; Allekotte, I.; Antico, F.; Antonelli, L.A.; et al. Design concepts for the Cherenkov Telescope Array CTA: An advanced facility for ground-based high-energy gamma-ray astronomy. Exp. Astron. 2011, 32, 193-316. [CrossRef] 
69. Abeysekara, A.U.; Alfaro, R.; Alvarez, C.; Álvarez, J.D.; Arceo, R.; Arteaga-Velázquez, J.C.; Rojas, A.D.; Solares, H.A.A.; Barber, A.S. The HAWC Real-time Flare Monitor for Rapid Detection of Transient Events. Astrophys. J. 2017, 843, 116. [CrossRef]

70. Abdollahi, S.; Ackermann, M.; Ajello, M.; Albert, A.; Baldini, L.; Ballet, J.; Barbiellini, G.; Bastieri, D.; Gonzalez, B.J. The Second Catalog of Flaring Gamma-Ray Sources from the Fermi All-sky Variability Analysis. Astrophys. J. 2017, 846, 34. [CrossRef]

(C) 2018 by the author. Licensee MDPI, Basel, Switzerland. This article is an open access article distributed under the terms and conditions of the Creative Commons Attribution (CC BY) license (http:/ / creativecommons.org/licenses/by/4.0/). 